\title{
APPENDIX B: SAMPLE TELEMEDICAL PROTOCOLS
}

\section{Protocols for Emergencies}

Emergency situations can arise even from the simplest complaint. However, some complaints warrant a higher level of care than telemedicine can provide. These situations include chest pain, especially with a patient with comorbidities, abdominal pain with fever, and shortness of breath, among other concerns.

Protocols for dealing with these situations should include keeping the patient on the video chat, having a family member or staff member call 911, keeping the patient calm, and getting information together for the receiving hospital. In certain cases, such as chest pain, advising the patient take baby aspirin may be indicated. Any intervention performed should be detailed to the 911 dispatcher and any first responders that are available: EMS, police, fire/rescue.

\section{Protocols for COVID-19 (Infectious Disease)}

All practices should have an infectious disease screening tool in place. During the COVID-19 pandemic, my practice created a flow sheet to guide call center staff and receptionists in the 
proper means to screen patients for appropriateness of being seen in-office versus digitally. Initially, this screening tool contained travel history but that was phased out as the pandemic went on. Questions that did not change included symptoms: fever, sore throat, cough, congestion, and shortness of breath. Known exposure was also an important diagnostic factor in the telephone triage assessment for evaluation of the patient. Again, later in the pandemic this became less of a criterion as the transmission became more skewed and patients were found to contract the disease without known contact with an infected person.

\section{General Protocols}

Overall, the patient should be screened for appropriateness. Depending on the state, a previous relationship may be required to be established prior to this visit. The call center or office receptionist should be aware of the nature of the pending telemedical visit. This will help to avoid frustration and confusion should the provider not be able to meet the needs of the patient (e.g., refill of controlled medications or a complaint that cannot meet the standard of care via telemedicine). 